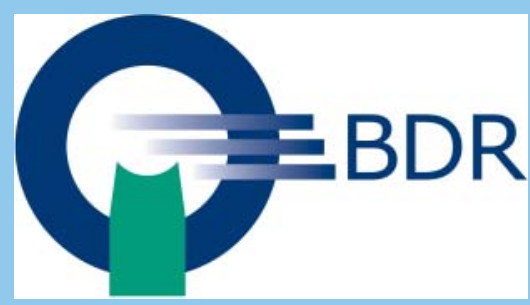

\title{
RöKo 2017 - Radiologie in Klinik und Praxis
}

Die in weiten Teilen vom BDR organisierte Reihe „Radiologie in Klinik und Praxis“ ist weiter gewachsen und nun ein stabiler und gut besuchter Programmpunkt des Röntgenkongresses geworden.

In der Veranstaltung III referierte Prof. Dr. Dr. Thomas Ufer, Rechtsanwalt und Arzt, Fachanwalt für Medizinrecht, Hamburg, zum Thema „Ist der §299a StGB ein Damoklesschwert auch für Radiologen?“ und fasste seinen Vortrag in den Kernaussagen zusammen.

„Ob das Gesetz zur Bekämpfung von Korruption im Gesundheitswesen, durch welches bestimmte Verhaltensweisen auch strafrechtlich sanktioniert werden, notwendig war oder nicht, ist auch weiterhin umstritten. Weitgehende Einigkeit besteht aber darüber, dass mit dem Gesetz eine erhebliche Verunsicherung eingetreten ist, auch und gerade soweit Kooperationen zwischen Leistungserbringern betroffen sind. Diese Verunsicherung betrifft insbesondere kooperationsaffine Fachgebiete wie die Radiologie, wo ein originäres Patientenklientel nicht besteht, sondern - geradezu zwangsläufig - Zuweisungen durch andere erfolgen (müssen).

Die besondere Schwierigkeit in der Rechtsanwendung liegt dabei in einer trennscharfen Abgrenzung zwischen (erlaubten) Kooperationen und (verbotener) Korruption. Während nämlich anhand von Trennungs-, Transparenz-, Dokumentations- und Äquivalenzprinzip versucht wird, eine Abgrenzung zwischen legalen und illegalen Verhaltensweisen zu erreichen, besteht oftmals aus der ex-ante-Perspektive eine geringe Trennschärfe. Dies wird noch durch die Breitenwirkung von Strafverfolgungsmaßnahmen erhöht, da Strafverfolgungsbehörden - etwa bei (anonymen) Anzeigen geradezu gezwungen sind, Ermittlungen aufzunehmen, um eine Tatsachengrundlage ob der Überprüfung einer Strafbarkeit zu schaffen. Hierbei vorzunehmende Abwägungen, insbesondere zur Angemessenheit von Vergütungen, bleiben dabei oft wertungsoffen, sowohl was eine Angemessenheit „im weiteren Sinne“ angeht, also hinsichtlich der Festlegung des manifesten ökonomischen und der latenten Nutzen im Einzelfall, aber auch die Angemessenheit einer Vergütung für die Arbeitskraft des Arztes („Angemessenheit im engeren Sinne“).

Diese notwendige Feststellung der Angemessenheit lässt sich auf jegliche Vereinbarung für Kooperationen im Gesundheitswesen übertragen, beginnend von dem Abschluss eines Mietvertrages für Praxisräumlichkeiten zwischen unterschiedlichen Beteiligten über ambulant-stationäre Kooperationsmodelle bis hin zur konkreten Preisgestaltung im Rahmen von Einkaufsbzw. Abgabepreisen, etwa für Kontrastmittel. Bisherige Versuche, hierfür Leitplanken festzulegen, befinden sich bislang noch im Anfangsstadium und finden keine unumstrittene Resonanz.

Insoweit kann es dem Arzt zum jetzigen Zeitpunkt nur angeraten werden, in seinem Einflussbereich eine Bestandsaufnahme vorzunehmen über in der Vergangenheit eingegangene vertragliche Verpflichtungen. So- bald eine Motivationslage hervortritt, die eine Kick-Back-Konstellation jedenfalls nahelegen könnte, besteht in jedem Falle weiterer Abklärungsbedarf und ggf. die Notwendigkeit, Änderungen an den eigenen Verhaltensweisen vorzunehmen. Gibt es für die Überprüfung von Verhaltensweisen Clearingstellen und/oder Aufsichtsbehörden, so kann der Gang zu diesen nur angeraten werden, wenn dies nicht - wie etwa in bestimmten Bundesländern (z. B. Ärztekammer Niedersachsen) - sowieso Berufspflicht ist. Leider sieht das Strafprozessrecht eine Vorabanfrage an die für die Korruptionsstrafbarkeit zuständigen Staatsanwaltschaften nicht vor, so dass durch diese keine verbindliche Festlegung im Vorfeld eines Ermittlungsverfahrens erreicht werden kann.

Entsprechende Abwägungen können immer nur im jeweiligen Einzelfall getroffen werden; die Einführung einer Korruptionsstrafbarkeit mag aber jedenfalls den Anlass bilden, mit korruptionsgefährdeten Konstellationen heute noch sensibler umzugehen als in der Vergangenheit."

Die Zusammenfassungen der übrigen Vorträge aus dieser Ausgaben finden Sie in den Juliund August-Ausgaben von DER RADIOLOGE.

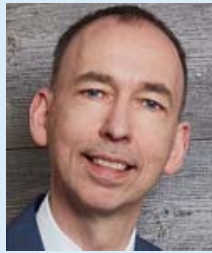

Prof. Dr. Dr. Thomas Ufer 